\title{
CONVERGENCE OF CESÁRO MEANS WITH VARYING PARAMETERS OF WALSH-FOURIER SERIES
}

\author{
ANAS AHMAD ABU JOUDEH AND GYÖRGY GÁT
}

Received 06 June, 2017

\begin{abstract}
In 2007 Akhobadze [1] (see also [2]) introduced the notion of Cesàro means of Fourier series with variable parameters. In the present paper we prove the almost everywhere convergence of the the Cesàro $\left(C, \alpha_{n}\right)$ means of integrable functions $\sigma_{n}^{\alpha_{n}} f \rightarrow f$, where $\mathbb{N}_{\alpha, K} \ni n \rightarrow$ $\infty$ for $f \in L^{1}(I)$, where $I$ is the Walsh group for every sequence $\alpha=\left(\alpha_{n}\right), 0<\alpha_{n}<1$. This theorem for constant sequences $\alpha$ that is, $\alpha \equiv \alpha_{1}$ was proved by Fine [3].

2010 Mathematics Subject Classification: 42C10
\end{abstract}

\section{INTRODUCTION AND MAIN RESULTS}

We follow the standard notions of dyadic analysis introduced by the mathematicians F. Schipp, P. Simon, W. R. Wade (see e.g. [9]) and others. Denote by $\mathbb{N}:=\{0,1, \ldots\}, \mathbb{P}:=\mathbb{N} \backslash\{0\}$, the set of natural numbers, the set of positive integers and $I:=[0,1)$ the unit interval. Denote by $\lambda(B)=|B|$ the Lebesgue measure of the set $B(B \subset I)$. Denote by $L^{p}(I)$ the usual Lebesgue spaces and $\|\cdot\|_{p}$ the corresponding norms $(1 \leq p \leq \infty)$. Set

$$
\mathcal{g}:=\left\{\left[\frac{p}{2^{n}}, \frac{p+1}{2^{n}}\right\}: p, n \in \mathbb{N}\right\}
$$

the set of dyadic intervals and for given $x \in I$ and let $I_{n}(x)$ denote the interval $I_{n}(x) \in \mathcal{g}$ of length $2^{-n}$ which contains $x(n \in \mathbb{N})$. Also use the notion $I_{n}:=$ $I_{n}(0)(n \in \mathbb{N})$. Let

$$
x=\sum_{n=0}^{\infty} x_{n} 2^{-(n+1)}
$$

be the dyadic expansion of $x \in I$, where $x_{n}=0$ or 1 and if $x$ is a dyadic rational number $\left(x \in\left\{\frac{p}{2^{n}}: p, n \in \mathbb{N}\right\}\right)$ we choose the expansion which terminates in 0 's. The

Research is supported by the Hungarian National Foundation for Scientific Research (OTKA), grant no. K111651 and by project EFOP-3.6.1-16-2016-00022 supported by the European Union, co-financed by the European Social Fund. 
notion of the Hardy space $H(I)$ is introduced in the following way [9]. A function $a \in L^{\infty}(I)$ is called an atom, if either $a=1$ or a has the following properties: supp $a \subseteq I_{a},\|a\|_{\infty} \leq\left|I_{a}\right|^{-1}, \int_{I} a=0$, for some $I_{a} \in \mathcal{g}$. We say that the function $f$ belongs to $H$, if $f$ can be represented as $f=\sum_{i=0}^{\infty} \lambda_{i} a_{i}$, where $a_{i}$ 's are atoms and for the coefficients $\left(\lambda_{i}\right)$ the inequality $\sum_{i=0}^{\infty}\left|\lambda_{i}\right|<\infty$ is true. It is known that $H$ is a Banach space with respect to the norm

$$
\|f\|_{H}:=\inf \sum_{i=0}^{\infty}\left|\lambda_{i}\right|
$$

where the infimum is taken over all decompositions $f=\sum_{i=0}^{\infty} \lambda_{i} a_{i} \in H$.

Set the definition of the $n$th $(n \in \mathbb{N})$ Walsh-Paley function at point $x \in I$ as:

$$
\omega_{n}(x):=\prod_{j=0}^{\infty}(-1)^{x_{j} n_{j}},
$$

where $\mathbb{N} \ni n=\sum_{n=0}^{\infty} n_{j} 2^{j}\left(n_{j} \in\{0,1\}(j \in \mathbb{N})\right)$. It is known (see [8] or [10]) that the system $\left(\omega_{n}, n \in \mathbb{N}\right)$ is the character system of $(I,+)$, where the group operation + is the so-called dyadic or logical addition on $I$. That is, for any $x, y \in I$

$$
x+y:=\sum_{n=0}^{\infty}\left|x_{n}-y_{n}\right| 2^{-(n+1)} .
$$

Denote by

$$
\hat{f}(n):=\int_{I} f \omega_{n} d \lambda, \quad D_{n}:=\sum_{k=0}^{n-1} \omega_{k}, \quad K_{n}^{1}:=\frac{1}{n+1} \sum_{k=0}^{n} D_{k}
$$

the Fourier coefficients, the Dirichlet and the Fejér or $(C, 1)$ kernels, respectively. It is also known that the Fejér or $(C, 1)$ means of $f$ is

$$
\begin{gathered}
\sigma_{n}^{1} f(y):=\frac{1}{n+1} \sum_{k=0}^{n} S_{k} f(y)=\int_{I} f(x) K_{n}^{1}(y+x) d \lambda(x) \\
=\frac{1}{n+1} \sum_{k=0}^{n} \int_{I} f(x) D_{k}(y+x) d \lambda(x), \quad(n \in \mathbb{N}, y \in I) .
\end{gathered}
$$

It is known [9] that for $n \in \mathbb{N}, x \in I$ it holds

$$
D_{2^{n}}(x)=\left\{\begin{array}{l}
2^{n}, \text { if } x \in I_{n} \\
0, \text { if } x \notin I_{n}
\end{array}\right.
$$

and also that

$$
D_{n}(x)=\omega_{n}(x) \sum_{k=1}^{\infty} D_{2^{k}}(x) n_{k}(-1)^{x_{k}},
$$


where $n=\sum_{i=1}^{\infty} n_{i} 2^{i}, n_{i}=\{0,1\}(i \in \mathbb{N})$.

Denote by $K_{n}^{\alpha_{n}}$ the kernel of the summability method $\left(C, \alpha_{n}\right)$ and call it the $\left(C, \alpha_{n}\right)$ kernel or the Cesàro kernel for $\alpha_{n} \in \mathbb{R} \backslash\{-1,-2, \ldots\}$

$$
K_{n}^{\alpha_{n}}=\frac{1}{A_{n}^{\alpha_{n}}} \sum_{k=0}^{n} A_{n-k}^{\alpha_{n}-1} D_{k}
$$

where

$$
A_{k}^{\alpha_{n}}=\frac{\left(\alpha_{n}+1\right)\left(\alpha_{n}+2\right) \ldots\left(\alpha_{n}+n\right)}{k !} .
$$

It is known [12] that $A_{n}^{\alpha_{n}}=\sum_{k=0}^{n} A_{k}^{\alpha_{n}-1}, A_{k}^{\alpha_{n}}-A_{k+1}^{\alpha_{n}}=-\frac{\alpha_{n} A_{k}^{\alpha_{n}}}{k+1}$. The $\left(C, \alpha_{n}\right)$ Cesàro means of integrable function $f$ is

$$
\sigma_{n}^{\alpha_{n}} f(y):=\frac{1}{A_{n}^{\alpha_{n}}} \sum_{k=0}^{n} A_{n-k}^{\alpha_{n}-1} S_{k} f(y)=\int_{I} f(x) K_{n}^{\alpha_{n}}(y+x) d \lambda(x) .
$$

In [3] Fine proved the almost everywhere convergence $\sigma_{n}^{\alpha_{n}} f \longrightarrow f$ for all integrable function $f$ with constant sequence $\alpha_{n}=\alpha_{1}>0$. On the rate of convergence of Cesàro means in this constant case see the paper of Fridli [4]. For the two-dimensional situation see the paper of Goginava [7].

Comment 1 . With respect to other locally constant orthonormal sysytems for instance it was a question of Taibleson [8] open for a long time, that does the FejérLebesgue theorem, that is the a.e. convergence $\sigma_{n}^{1} f \longrightarrow f$ hold for all integrable function $f$ with respect to the character system of the group of 2-adic integers. In 1997 Gát answered [1] this question in the affirmative. Zheng and Gát generalized this result $[9,2]$ for more general orthonormal systems.

Set two variable function $P(n, \alpha):=\sum_{i=0}^{\infty} n_{i} 2^{i \alpha}$ for $n \in \mathbb{N}, \alpha \in \mathbb{R}$. For instance $P(n, 1)=n$. Also set for sequences $\alpha=\left(\alpha_{n}\right)$ and positive reals $K$ the subset of natural numbers

$$
\mathbb{N}_{\alpha, K}:=\left\{n \in \mathbb{N}: \frac{P\left(n, \alpha_{n}\right)}{n^{\alpha_{n}}} \leq K\right\} .
$$

We can easily remark that for a sequence $\alpha$ such that $1>\alpha_{n} \geq \alpha_{0}>0$ we have $\mathbb{N}_{\alpha, K}=\mathbb{N}$ for some $K$ depending only on $\alpha_{0}$. We also remark that $2^{n} \in \mathbb{N}_{\alpha, K}$ for every $\alpha=\left(\alpha_{n}\right), 0<\alpha_{n}<1$ and $K \geq 1$.

In this paper $C$ denotes an absolute constant and $C_{K}$ another one which may depend only on $K$. The introduction of $\left(C, \alpha_{n}\right)$ means due to Akhobadze investigated [1] the behavior of the $L^{1}$-norm convergence of $\sigma_{n}^{\alpha_{n}} f \rightarrow f$ for the trigonometric system. In this paper it is also supposed that $1>\alpha_{n}>0$ for all $n$.

The main aim of this paper is to prove :

Theorem 1. Suppose that $1>\alpha_{n}>0$. Let $f \in L^{1}(I)$. Then we have the almost everywhere convergence $\sigma_{n}^{\alpha_{n}} f \rightarrow f$ provided that $\mathbb{N}_{\alpha, K} \ni n \rightarrow \infty$. 
The method we use to prove Theorem 1 is to investigate the maximal operator $\sigma_{*}^{\alpha} f:=\sup _{n \in \mathbb{N}_{\alpha, K}}\left|\sigma_{n}^{\alpha_{n}} f\right|$. We also prove that this operator is a kind of type $(H, L)$ and of type $\left(L^{p}, L^{p}\right)$ for all $1<p \leq \infty$. That is,

Theorem 2. Suppose that $1>\alpha_{n}>0$. Let $|f| \in H(I)$. Then we have

$$
\left\|\sigma_{*}^{\alpha} f\right\|_{1} \leq C_{K}\||f|\|_{H} .
$$

Moreover, the operator $\sigma_{*}^{\alpha}$ is of type $\left(L^{p}, L^{p}\right)$ for all $1<p \leq \infty$. That is,

$$
\left\|\sigma_{*}^{\alpha} f\right\|_{p} \leq C_{K, p}\|f\|_{p}
$$

for all $1<p \leq \infty$.

For the sequence $\alpha_{n}=1$ Theorem 2 is due to Fujii [5]. For the more general but constant sequence $\alpha_{n}=\alpha_{1}$ see Weisz [11].

Basically, in order to prove Theorem 1 we verify that the maximal operator $\sigma_{*}^{\alpha} f\left(\alpha=\left(\alpha_{n}\right)\right)$ is of weak type $\left(L^{1}, L^{1}\right)$. The way we get this, the investigation of kernel functions, and its maximal function on the unit interval $I$ by making a hole around zero and some quasi locality issues (for the notion of quasi-locality see [9]). To have the proof of Theorem 2 is the standard way. We need several Lemmas in the next section.

\section{Proofs}

Lemma 1. For $j, n \in \mathbb{N}, j<2^{n}$ we have

$$
D_{2^{n}-j}(x)=D_{2^{n}}(x)-\omega_{2^{n}-1}(x) D_{j}(x) .
$$

Proof.

$$
D_{2^{n}}(x)=\sum_{k=0}^{2^{n}-1} \omega_{k}(x)=\sum_{k=0}^{2^{n}-j-1} \omega_{k}(x)+\sum_{k=2^{n}-j}^{2^{n}-1} \omega_{k}(x)=D_{2^{n}-j}+\sum_{k=2^{n}-j}^{2^{n}-1} \omega_{k}(x) .
$$

We have to prove :

$$
\sum_{k=2^{n}-j}^{2^{n}-1} \omega_{k}(x)=\omega_{2^{n}-1}(x) D_{j}(x)
$$

For $k<j, k=k_{n-1} 2^{n-1}+\ldots+k_{1} 2^{1}+k_{0}$ we have

$$
\begin{aligned}
& \omega_{2^{n}-1}(x) \omega_{k} \\
& =\omega_{2^{n-1}+\ldots+2^{1}+2^{0}}(x) \omega_{k_{n-1} 2^{n-1}+\ldots+k_{0}}(x) \\
& =\omega_{\left(1+k_{n-1}(\bmod 2)\right) 2^{n-1}+\ldots+\left(1+k_{0}(\bmod 2)\right) 2^{0}(x)} \\
& =\omega_{\left(1-k_{n-1}(\bmod 2)\right) 2^{n-1}+\ldots+\left(1-k_{0}(\bmod 2)\right) 2^{0}(x)} \\
& =\omega_{2^{n-1}+2^{n-2}+\ldots+2^{0}-\left(k_{n-1} 2^{n-1}+\ldots+k_{0}\right)}(x)=\omega_{2^{n}-1-k}(x) \text {. }
\end{aligned}
$$


Thus,

$$
\omega_{2^{n}-1}(x) D_{j}(x)=\omega_{2^{n}-1}(x) \sum_{k=0}^{j-1} \omega_{k}(x)=\sum_{k=0}^{j-1} \omega_{2^{n}-1-k}(x)=\sum_{k=2^{n}-j}^{2^{n}-1} \omega_{k}(x) .
$$

This completes the proof of Lemma 1.

Introduce the following notations: for $a, n, j \in \mathbb{N}$ let $n_{(j)}:=\sum_{i=0}^{j-1} n_{i} 2^{i}$, that is, $n_{(0)}=0, n_{(1)}=n_{0}$ and for $2^{B} \leq n<2^{B+1}$, let $|n|:=B, n=n_{(B+1)}$. Moreover, introduce the following functions and operators for $n \in \mathbb{N}$ and $1>\alpha_{n}>0$

$$
\begin{aligned}
& T_{n}^{\alpha_{a}}:= \frac{1}{A_{n}^{\alpha_{a}}} \sum_{j=0}^{2^{|n|}-1} A_{n-j}^{\alpha_{a}-1} D_{j}, \\
& \tilde{T}_{n}^{\alpha_{a}}:=\frac{1}{A_{n}^{\alpha_{a}}} D_{2^{B}} \sum_{j=0}^{2^{B}-1} A_{n_{(B)}+j}^{\alpha_{a}-1} \\
& \quad+\left(1-\alpha_{a}\right) \sum_{j=0}^{2^{B}-1} A_{n_{(B)}+j}^{\alpha_{a}-1} \frac{j+1}{n_{(B)}+j+1}\left|K_{j}^{1}\right|+A_{n}^{\alpha_{a}-1} 2^{B}\left|K_{2^{B}-1}^{1}\right|, \\
& t_{n}^{\alpha_{a}} f(y):=\int_{I} f(x) T_{n}^{\alpha_{a}}(y+x) d \lambda(x), \\
& \tilde{t}_{n}^{\alpha_{a}} f(y):=\int_{I} f(x) \tilde{T}_{n}^{\alpha_{a}}(y+x) d \lambda(x) .
\end{aligned}
$$

Now, we need to prove the next Lemma which means that maximal operator $\sup _{n, a}\left|\tilde{t}_{n}^{\alpha}\right|$ is quasi-local. This lemma together with the next one are the most important tools in the proof of the main results of this paper.

Lemma 2. Let $1>\alpha_{a}>0, f \in L^{1}(I)$ such that supp $f \subset I_{k}(u), \int_{I_{k}(u)} f d \lambda=0$ for some dyadic interval $I_{k}(u)$. Then we have

$$
\int_{I \backslash I_{k}(u)} \sup _{n, a \in \mathbb{N}}\left|\tilde{t}_{n}^{\alpha_{a}} f\right| d \lambda \leq C\|f\|_{1} .
$$

Moreover, $\left|T_{n}^{\alpha_{a}}\right| \leq \tilde{T}_{n}^{\alpha_{a}}$.

Proof. It is easy to have that for $n<2^{k}$ and $x \in I_{k}(u)$ we have $\tilde{T}_{n}^{\alpha_{a}}(y+x)=$ $\tilde{T}_{n}^{\alpha_{a}}(y+u)$ and

$$
\begin{aligned}
& \int_{I_{k}(u)} f(x) \tilde{T}_{n}^{\alpha_{a}}(y+x) d \lambda(x) \\
& =\tilde{T}_{n}^{\alpha_{a}}(y+u) \int_{I_{k}(u)} f(x) d \lambda(x)=0 .
\end{aligned}
$$


Therefore,

$$
\int_{I \backslash I_{k}(u)} \sup _{n, a \in \mathbb{N}} \tilde{t}_{n}^{\alpha_{a}} f d \lambda=\int_{I \backslash I_{k}(u)_{n \geq 2^{k}, a \in \mathbb{N}}} \sup _{n} \tilde{t}_{n}^{\alpha_{a}} f d \lambda .
$$

Recall that $B=|n|$. Then

$$
\begin{aligned}
& A_{n}^{\alpha_{a}} T_{n}^{\alpha_{a}} \\
& =\sum_{j=0}^{2^{B}-1} A_{2^{B}+n_{(B)}-j}^{\alpha_{a}-1} D_{j} \\
& =\sum_{j=0}^{2^{B}-1} A_{n_{(B)}+j}^{\alpha_{a}-1} D_{2^{B}-j}
\end{aligned}
$$

By Lemma 1 we have

$$
\begin{aligned}
& A_{n}^{\alpha_{a}} T_{n}^{\alpha_{a}} \\
& =D_{2^{B}} \sum_{j=0}^{2^{B}-1} A_{n_{(B)}+j}^{\alpha_{a}-1}-\omega_{2^{B}-1} \sum_{j=0}^{2^{B}-1} A_{n_{(B)}+j}^{\alpha_{a}-1} D_{j} .
\end{aligned}
$$

It is easy to have that $\frac{1}{A_{n}^{\alpha a}} D_{2^{B}}(z) \sum_{j=0}^{2^{B}-1} A_{n_{(B)}+j}^{\alpha_{a}-1}=0$, for any $z \in I \backslash I_{k}$. This holds because $D_{2^{B}}(z)=0$ for $B=|n| \geq k$ and $z \in I \backslash I_{k}$. By the help of the Abel transform we get: 


$$
\begin{aligned}
& \left|\sum_{j=0}^{2^{B}-1} A_{n_{(B)}+j}^{\alpha_{a}-1} D_{j}\right| \\
& =\mid \sum_{j=0}^{2^{B}-1}\left(A_{n_{(B)}+j}^{\alpha_{a}-1}-A_{n_{(B)}+j+1}^{\alpha_{a}-1} \sum_{i=0}^{j} D_{i}+A_{n_{(B)}+2^{B}}^{\alpha_{a}-1} \sum_{i=0}^{2^{B}-1} D_{i} \mid\right. \\
& =\left|\left(1-\alpha_{a}\right) \sum_{j=0}^{2^{B}-1} A_{n_{(B)}+j}^{\alpha_{a}-1} \frac{j+1}{n_{(B)}+j+1} K_{j}^{1}+A_{n}^{\alpha_{a}-1} 2^{B} K_{2^{B}-1}^{1}\right| \\
& =\mid\left(1-\alpha_{a}\right) \sum_{j=0}^{2^{k}-1} A_{n_{(B)}+j}^{\alpha_{a}-1} \frac{j+1}{n_{(B)}+j+1} K_{j}^{1}+\left(1-\alpha_{a}\right) \sum_{j=2^{k}}^{2^{B}-1} A_{n_{(B)}+j}^{\alpha_{a}-1} \frac{j+1}{n_{(B)}+j+1} K_{j}^{1} \\
& +A_{n}^{\alpha_{a}-1} 2^{B} K_{2^{B}-1}^{1} \mid \\
& \leq\left(1-\alpha_{a}\right) \sum_{j=0}^{2^{k}-1} A_{n_{(B)}+j}^{\alpha_{a}-1} \frac{j+1}{n_{(B)}+j+1}\left|K_{j}^{1}\right| \\
& +\left(1-\alpha_{a}\right) \sum_{j=2^{k}}^{2^{B}-1} A_{n_{(B)}+j}^{\alpha_{a}-1} \frac{j+1}{n_{(B)}+j+1}\left|K_{j}^{1}\right|+A_{n}^{\alpha_{a}-1} 2^{B}\left|K_{2^{B}-1}^{1}\right| \\
& =: I+I I+I I I .
\end{aligned}
$$

These equalities above immediately proves inequality $\left|T_{n}^{\alpha_{a}}\right| \leq \tilde{T}_{n}^{\alpha_{a}}$.

Since for any $j<2^{k}$ we have that the Fejér kernel $K_{j}^{1}(y+x)$ depends (with respect to $x$ ) only on coordinates $x_{0}, \ldots, x_{k-1}$, then $\int_{I_{k}(u)} f(x)\left|K_{j}^{1}(y+x)\right| d \lambda(x)=$ $\left|K_{j}^{1}(y+u)\right| \int_{I_{k}(u)} f(x) d \lambda(x)=0$ gives $\int_{I_{k}(u)} f(x) I(y+x) d \lambda(x)=0$.

On the other hand,

$$
\begin{aligned}
& I I=\left(1-\alpha_{a}\right) \sum_{j=2^{k}}^{2^{B}-1} A_{n_{(B)}+j}^{\alpha_{a}-1} \frac{j+1}{n_{(B)}+j+1}\left|K_{j}^{1}\right| \\
& \leq \sup _{j \geq 2^{k}}\left|K_{j}^{1}\right|\left(1-\alpha_{a}\right) \sum_{j=0}^{n} A_{j}^{\alpha_{a}-1}=A_{n}^{\alpha_{a}}\left(1-\alpha_{a}\right) \sup _{j \geq 2^{k}}\left|K_{j}^{1}\right| .
\end{aligned}
$$

This by Lemma 3 in [6] gives

$$
\int_{I \backslash I_{k}} \sup _{n \geq 2^{k}, a \in \mathbb{N}} \frac{1}{A_{n}^{\alpha_{a}}} I I d \lambda \leq \int_{I \backslash I_{k}} \sup _{j \geq 2^{k}}\left|K_{j}^{1}\right| d \lambda \leq C .
$$


The situation with III is similar. Namely,

$$
\frac{A_{n}^{\alpha_{a}-1} n}{A_{n}^{\alpha_{a}}}=\frac{\alpha_{a} \cdot n}{\left(\alpha_{a}+n\right)} \leq \alpha_{a}<1 .
$$

So, just as in the case of $I I$ we apply Lemma 3 in [6]

$$
\int_{I \backslash I_{k}} \sup _{n \geq 2^{k}, a \in \mathbb{N}} \frac{1}{A_{n}^{\alpha_{a}}} I I I d \lambda \leq \int_{I \backslash I_{k}} \sup _{n \geq 2^{k}, a \in \mathbb{N}}\left|K_{2^{|n|-1}}^{1}\right| d \lambda \leq C .
$$

Therefore, substituting $z=x+y \in I \backslash I_{k}$ (since $x \in I_{k}(u)$ and $y \in I \backslash I_{k}(u)$ )

$$
\begin{aligned}
& \int_{I \backslash I_{k}(u)} \sup _{n \geq 2^{k}, a \in \mathbb{N}} \tilde{t}_{n}^{\alpha_{a}} f d \lambda \\
& =\int_{I \backslash I_{k}(u)} \sup _{n \geq 2^{k}, a \in \mathbb{N}}\left|\int_{I_{k}(u)} f(x) \tilde{T}_{n}^{\alpha_{a}}(y+x) d \lambda(x)\right| d \lambda(y) \\
& \leq \int_{I \backslash I_{k}(u)} \int_{I_{k}(u)}|f(x)| \sup _{n \geq 2^{k}, a \in \mathbb{N}} \frac{1}{A_{n}^{\alpha_{a}}}(I I(y+x)+I I I(y+x)) d \lambda(x) \\
& =\int_{I_{k}(u)}|f(x)| \int_{I \backslash I_{k}} \sup _{n \geq 2^{k}, a \in \mathbb{N}} \frac{1}{A_{n}^{\alpha_{a}}}(I I(z)+I I I(z)) d \lambda(z) d \lambda(x) \\
& \leq C \int_{I_{k}(u)}|f(x)| d \lambda(x) .
\end{aligned}
$$

This completes the proof of Lemma 2.

A straightforward corollary of this lemma is:

Corollary 1. Let $1>\alpha_{a}>0$. Then we have $\left\|T_{n}^{\alpha_{a}}\right\|_{1} \leq\left\|\tilde{T}_{n}^{\alpha_{a}}\right\|_{1} \leq C,\left\|t_{n}^{\alpha_{a}} f\right\|_{1}$, $\left\|\tilde{t}_{n}^{\alpha_{a}} f\right\|_{1} \leq C\|f\|_{1}$ and $\left\|t_{n}^{\alpha_{a}} g\right\|_{\infty},\left\|\tilde{t}_{n}^{\alpha_{a}} g\right\|_{\infty} \leq C\|g\|_{\infty}$ for all natural numbers $a, n$, where $C$ is some absolute constant and $f \in L^{1}, g \in L^{\infty}$. That is, operators $\tilde{t}_{n}^{\alpha_{a}}, t_{n}^{\alpha_{a}}$ is of type $\left(L^{1}, L^{1}\right)$ and $\left(L^{\infty}, L^{\infty}\right)$ (uniformly in $n$ ).

Proof. The proof is a straightforward consequence of Lemma 2 and an easy estimation below. Let $B=|n|$. Then

$$
\begin{aligned}
& \left\|A_{n}^{\alpha_{a}} \tilde{T}_{n}^{\alpha_{a}}\right\|_{1} \leq\left\|D_{2^{B}}\right\|_{1} \sum_{j=0}^{2^{B}-1} A_{n_{(B)}+j}^{\alpha_{a}-1} \\
& +\left(1-\alpha_{a}\right) \sum_{j=0}^{2^{B}-1} A_{n_{(B)}+j}^{\alpha_{a}-1} \frac{j+1}{n_{(B)}+j+1}\left\|K_{j}^{1}\right\|_{1}+A_{n}^{\alpha_{a}-1} 2^{B}\left\|K_{2^{B}-1}^{1}\right\|_{1} .
\end{aligned}
$$

Then by $\left\|D_{2^{B}}\right\|_{1}=1,\left\|K_{j}^{1}\right\|_{1} \leq C$ we complete the proof of Corollary 1 . 
Lemma 3. Let $n, N$ be any natural numbers and $0<\alpha<1$. Then we have

$$
\frac{A_{n}^{\alpha}}{A_{N}^{\alpha}} \leq 2\left(\frac{n+1}{N}\right)^{\alpha}
$$

Proof. By definition we have

$$
\frac{A_{n}^{\alpha}}{A_{N}^{\alpha}}=\left(1-\frac{\alpha}{n+1+\alpha}\right) \cdots\left(1-\frac{\alpha}{N+\alpha}\right) \leq\left(1-\frac{\alpha}{n+2}\right) \cdots\left(1-\frac{\alpha}{N+1}\right) .
$$

It is well-known that

$$
\left(1-\frac{\alpha}{i(n+1)+1}\right) \cdots\left(1-\frac{\alpha}{(i+1)(n+1)}\right) \leq\left(1-\frac{\alpha}{(i+1)(n+1)}\right)^{n+1} \leq\left(e^{-1}\right)^{\frac{\alpha}{i+1}}
$$

for every $n \in \mathbb{N}$. This gives

$$
\begin{aligned}
& \left(1-\frac{\alpha}{n+2}\right) \cdots\left(1-\frac{\alpha}{N+1}\right) \leq\left(e^{-1}\right)^{\alpha \sum_{i=2}^{\left\lfloor\frac{N}{n+1}\right\rfloor} \frac{1}{i}} \\
& \leq\left(e^{-1}\right)^{\alpha \log _{e}\left\lfloor\frac{N}{n+1}\right\rfloor-1+c} \\
& \leq 2\left(e^{-1}\right)^{\alpha \log _{e}\left(\frac{N}{n+1}\right)}=2\left(\frac{n+1}{N}\right)^{\alpha},
\end{aligned}
$$

where $c \approx 0.5772$ is the Euler-Mascheroni constant. This completes the proof of Lemma 3.

Recall that the two variable function $P(n, \alpha)=\sum_{i=0}^{\infty} n_{i} 2^{i \alpha}$ for $n \in \mathbb{N}, \alpha \in \mathbb{R}$ and $K \in \mathbb{R}$ determines the set of natural numbers

$$
\mathbb{N}_{\alpha, K}=\left\{n \in \mathbb{N}: \frac{P\left(n, \alpha_{n}\right)}{n^{\alpha_{n}}} \leq K\right\} .
$$

Let $n=2^{h_{s}}+\cdots+2^{h_{0}}$, where $h_{s}>\cdots>h_{0} \geq 0$ are integers. That is, $|n|=h_{s}$. Let $n^{(j)}:=2^{h_{j}}+\cdots+2^{h_{0}}$. This means $n=n^{(s)}$. Define the following kernel function and operators

$$
\tilde{K}_{n}^{\alpha_{n}}:=\tilde{T}_{n^{(s)}}^{\alpha_{n}}+\sum_{l=0}^{s}\left(\frac{A_{n^{(l-1)}}^{\alpha_{n}}}{A_{n^{(s)}}^{\alpha_{n}}} D_{2^{h_{l}}}+\frac{A_{n^{(l-1)}}^{\alpha_{n}}}{A_{n^{(s)}}^{\alpha_{n}}} \tilde{T}_{n^{l-1}}^{\alpha_{n}}\right)
$$

and

$$
\tilde{\sigma}_{n}^{\alpha_{n}} f:=f * \tilde{K}_{n}^{\alpha_{n}}, \quad \tilde{\sigma}_{*}^{\alpha} f:=\sup _{n \in \mathbb{N}_{\alpha, K}}\left|f * \tilde{K}_{n}^{\alpha}\right| .
$$

In the sequel we prove that maximal operator $\tilde{\sigma}_{*}^{\alpha} f$ is quasi-local. This is the very base of the proof of the main results of this paper. That is, Theorem 1 and Theorem 2. 
Lemma 4. Let $1>\alpha_{n}>0, f \in L^{1}(I)$ such that supp $f \subset I_{k}(u), \int_{I_{k}(u)} f d \lambda=0$ for some dyadic interval $I_{k}(u)$. Then we have

$$
\int_{I \backslash I_{k}(u)} \tilde{\sigma}_{*}^{\alpha} f d \lambda \leq C_{K}\|f\|_{1},
$$

where constant $C_{K}$ can depend only on $K$.

Proof. Recall that $n=2^{h_{s}}+\cdots+2^{h_{0}}$, where $h_{s}>\cdots>h_{0} \geq 0$ are integers. That is, $|n|=h_{s}$. Let $n^{(j)}:=2^{h_{j}}+\cdots+2^{h_{0}}$. This means $n=n^{(s)}$. Use also the notation

$$
\begin{aligned}
& \tilde{K}_{n^{(s)}}^{\alpha_{n}} \\
& =\tilde{T}_{n^{(s)}}^{\alpha_{n}}+\sum_{l=0}^{s}\left(\frac{A_{n^{(l-1)}}^{\alpha_{n}}}{A_{n^{(s)}}^{\alpha_{n}}} D_{2^{h_{l}}}+\frac{A_{n^{(l-1)}}^{\alpha_{n}}}{A_{n^{(s)}}^{\alpha_{n}}} \tilde{T}_{n^{l-1}}^{\alpha_{n}}\right) \\
& =: G_{1}+G_{2}+G_{3} .
\end{aligned}
$$

Since $n^{(l-1)}<2^{h_{(l-1)}+1}$, then by Lemma 3 we have

$$
\frac{A_{n^{(l-1)}}^{\alpha_{n}}}{A_{n^{(s)}}^{\alpha_{n}}} \leq 2\left(\frac{n^{(l-1)}+1}{n^{(s)}}\right)^{\alpha_{n}} \leq 2 \frac{2^{\alpha_{n}\left(h_{l-1}+1\right)}}{2^{\alpha_{n} h_{s}}} \leq C \frac{2^{h_{l-1} \alpha_{n}}}{n^{\alpha_{n}}} .
$$

That is, by the above written we also have

$$
\begin{aligned}
& \int_{I \backslash I_{k}(u)} \sup _{n \in \mathbb{N}}\left|\int_{I_{k}(u)} f(x) G_{2}(y+x) d \lambda(x)\right| d \lambda(y) \\
& \int_{I \backslash I_{k}(u)} \sup _{n \in \mathbb{N}} \sum_{l=0}^{s} \frac{2^{h_{l-1} \alpha_{n}}}{n^{\alpha_{n}}}\left|\int_{I_{k}(u)} f(x) D_{2^{h}}(y+x) d \lambda(x)\right| d \lambda(y)=0
\end{aligned}
$$

since $f * D_{2^{h}}=0$ for $h \leq k$ because of the $\mathcal{A}_{k}$ measurability of $D_{2^{h}}$ and $\int f=0$. Besides, for $h>k D_{2^{h}}(y+x)=0\left(y+x \notin I_{k}\right)$.

As a result of these estimations above and by the help of Lemma 2, that is the quasi-locality of operator $\tilde{t}_{*}^{\alpha}=\sup _{n, a \in \mathbb{N}}\left|\tilde{t}_{n}^{\alpha_{a}}\right|$ we conclude

$$
\begin{aligned}
& \int_{I \backslash I_{k}(u)} \sup _{n \in \mathbb{N}}\left|\int_{I_{k}(u)} f(x)\left(G_{1}(y+x)+G_{3}(y+x)\right) d \lambda(x)\right| d \lambda(y) \\
& \leq C_{K} \int_{I \backslash I_{k}(u)} \sup _{n, a \in \mathbb{N}}\left|\int_{I_{k}(u)} f(x) \tilde{T}_{n}^{\alpha_{a}}(y+x) d \lambda(x)\right| d \lambda(y) \\
& \leq C_{K}\|f\|_{1} .
\end{aligned}
$$

This completes the proof of Lemma 4.

Lemma 5. The operator $\tilde{\sigma}_{*}^{\alpha}$ is of type $\left(L^{\infty}, L^{\infty}\right)\left(\tilde{\sigma}_{*}^{\alpha} f:=\sup _{n \in \mathbb{N}_{\alpha, K}}\left|\tilde{\sigma}_{n}^{\alpha_{n}} f\right|\right)$. 
Proof. By the help of the method of Lemma 4 and by Corollary 1 we have

$$
\begin{aligned}
& \left\|\tilde{K}_{n}^{\alpha_{n}}\right\|_{1}=\left\|\tilde{K}_{n^{(s)}}^{\alpha_{n}}\right\|_{1} \leq\left\|\tilde{T}_{n^{(s)}}^{\alpha_{n}}\right\|_{1}+\sum_{l=0}^{s}\left(\frac{A_{n^{(l-1)}}^{\alpha_{n}}}{A_{n^{(s)}}^{\alpha_{n}}}\left\|D_{2^{h_{l}}}\right\|_{1}+\frac{A_{n^{(l-1)}}^{\alpha_{n}}}{A_{n^{(s)}}^{\alpha_{n}}}\left\|\tilde{T}_{n^{l-1}}^{\alpha_{n}}\right\|_{1}\right) \\
& \leq C+C \sum_{l=0}^{s} \frac{A_{n^{(l-1)}}^{\alpha_{n}}}{A_{n^{(s)}}^{\alpha_{n}}} \leq C_{K}
\end{aligned}
$$

because $n \in \mathbb{N}_{\alpha, K}$. Hence $\tilde{\sigma}_{*}^{\alpha}$ is of type $\left(L^{\infty}, L^{\infty}\right)$ (with constant $\left.C_{K}\right)$. This completes the proof of Lemma 5 .

Now, we can prove the main tool in order to have Theorem 1 for operator $\sigma_{*}^{\alpha} f:=$ $\sup _{n \in \mathbb{N}_{\alpha, K}}\left|\sigma_{n}^{\alpha_{n}} f\right|$.

Lemma 6. The operators $\tilde{\sigma}_{*}^{\alpha}$ and $\sigma_{*}^{\alpha}$ are of weak type $\left(L^{1}, L^{1}\right)$.

Proof. First, we prove Lemma 6 for operator $\tilde{\sigma}_{*}^{\alpha}$. We apply the Calderon-Zygmund decomposition lemma [9]. That is, let $f \in L^{1}$ and $\|f\|_{1}<\delta$. Then there is a decomposition:

$$
f=f_{0}+\sum_{j=1}^{\infty} f_{j}
$$

such that $\left\|f_{0}\right\|_{\infty} \leq C \delta,\left\|f_{0}\right\|_{1} \leq C\|f\|_{1}$ and $I^{j}=I_{k_{j}}\left(u^{j}\right)$ are disjoint dyadic intervals for which

$$
\operatorname{supp} f_{j} \subset I^{j}, \int_{I^{j}} f_{j} d \lambda=0,|F| \leq \frac{C\left\|f_{1}\right\|}{\delta}
$$

$\left(u^{j} \in I, k_{j} \in N, j \in P\right.$ ), where $F=\cup_{i=1}^{\infty} I^{j}$. By the $\sigma$-sublinearity of the maximal operator with an appropriate constant $C_{K}$ we have

$$
\lambda\left(\tilde{\sigma}_{*}^{\alpha} f>2 C_{K} \delta\right) \leq \lambda\left(\tilde{\sigma}_{*}^{\alpha} f_{0}>C_{K} \delta\right)+\lambda\left(\tilde{\sigma}_{*}^{\alpha}\left(\sum_{i=1}^{\infty} f_{i}\right)>C_{K} \delta\right):=I+I I .
$$

Since by Lemma $5\left\|\tilde{\sigma}_{*}^{\alpha} f_{0}\right\|_{\infty} \leq C_{K}\left\|f_{0}\right\|_{\infty} \leq C_{K} \delta$ then we have $I=0$. So,

$$
\begin{aligned}
& \lambda\left(\tilde{\sigma}_{*}^{\alpha}\left(\sum_{i=1}^{\infty} f_{i}\right)>C_{K} \delta\right) \leq|F|+\lambda\left(\bar{F} \cap\left\{\tilde{\sigma}_{*}^{\alpha}\left(\sum_{i=1}^{\infty} f_{i}\right)>C_{K} \delta\right\}\right) \\
& \leq \frac{C_{K}\|f\|_{1}}{\delta}+\frac{C_{K}}{\delta} \sum_{i=1}^{\infty} \int_{I \backslash I^{j}} \tilde{\sigma}_{*}^{\alpha} f_{j} d \lambda=: \frac{C_{K}\|f\|_{1}}{\delta}+\frac{C_{K}}{\delta} \sum_{i=1}^{\infty} I I I_{j},
\end{aligned}
$$


where

$$
\begin{aligned}
I I I_{j} & :=\int_{I \backslash I^{j}} \tilde{\sigma}_{*}^{\alpha} f_{j} d \lambda \\
& \leq \int_{I \backslash I_{k_{j}}\left(u^{j}\right)} \sup _{n \in N_{\alpha, K}}\left|\int_{I_{k_{j}}\left(u^{j}\right)} f_{j}(x) \tilde{K}_{n}^{\alpha_{n}}(y+x) d \lambda(x)\right| d \lambda(y) .
\end{aligned}
$$

The forthcoming estimation of $I I I_{j}$ is given by the help Lemma 4

$$
I I I_{j} \leq C_{K}\left\|f_{j}\right\|_{1} .
$$

That is, operator $\tilde{\sigma}_{*}^{\alpha}$ is of weak type $\left(L^{1}, L^{1}\right)$. Next, we prove the estimation

$$
\left|K_{n}^{\alpha_{n}}\right| \leq \tilde{K}_{n}^{\alpha_{n}} .
$$

To prove (1) recall again that $n=2^{h_{s}}+\cdots+2^{h_{0}}$, where $h_{s}>\cdots>h_{0} \geq 0$ are integers. Since $n=2^{h_{s}}+n^{(s-1)}$, then we have

$$
\begin{aligned}
& \sum_{j=2^{h_{s}}}^{2^{h_{s}}+n^{(s-1)}} A_{n^{(s-1)}+2^{h_{s}-j}}^{\alpha_{n}-1} D_{j}=\sum_{k=0}^{n^{(s-1)}} A_{n^{(s-1)}-k}^{\alpha_{n}-1} D_{2^{h_{s}}+k} \\
= & D_{2^{h_{s}}} \sum_{k=0}^{n^{(s-1)}} A_{n^{(s-1)}-k}^{\alpha_{n}-1}+\omega_{2^{h_{s}}} \sum_{k=0}^{n^{(s-1)}} A_{n^{(s-1)}-k}^{\alpha_{n}-1} D_{k} \\
= & D_{2^{h_{s}}} A_{n^{(s-1)}}^{\alpha_{n}}+\omega_{2^{h_{s}}} A_{n^{(s-1)}}^{\alpha_{n}} K_{n^{(s-1)}}^{\alpha_{n}} .
\end{aligned}
$$

So, by the help of the equalities above we get

$$
K_{n^{(s)}}^{\alpha_{n}}=T_{n^{(s)}}^{\alpha_{n}}+\frac{A_{n^{(s-1)}}^{\alpha_{n}}}{A_{n^{(s)}}^{\alpha_{n}}}\left(D_{2^{h_{s}}}+\omega_{2^{h_{s}}} K_{n^{(s-1)}}^{\alpha_{n}}\right) .
$$

Apply this last formula recursively and Lemma 2. (Note that $n^{(-1)}=0, T_{0}^{\alpha_{n}}=$ $K_{0}^{\alpha_{n}}=0, A_{0}^{\alpha_{n}}=1$.)

$$
\begin{aligned}
& \left|K_{n}^{\alpha_{n}}\right|=\left|K_{n^{(s)}}^{\alpha_{n}}\right| \leq\left|T_{n^{(s)}}^{\alpha_{n}}\right|+\sum_{l=0}^{s}\left(\prod_{j=l}^{s} \frac{A_{n^{(j-1)}}^{\alpha_{n}}}{A_{n^{(j)}}^{\alpha_{n}}} D_{2^{h_{l}}}+\prod_{j=l}^{s} \frac{A_{n^{(j-1)}}^{\alpha_{n}}}{A_{n^{(j)}}^{\alpha_{n}}}\left|T_{n^{l-1}}^{\alpha_{n}}\right|\right) \\
& =\left|T_{n^{(s)}}^{\alpha_{n}}\right|+\sum_{l=0}^{s}\left(\frac{A_{n^{(l-1)}}^{\alpha_{n}}}{A_{n^{(s)}}^{\alpha_{n}}} D_{2^{h_{l}}}+\frac{A_{n^{(l-1)}}^{\alpha_{n}}}{A_{n^{(s)}}^{\alpha_{n}}}\left|T_{n^{l-1}}^{\alpha_{n}}\right|\right) \\
& \leq \tilde{K}_{n^{(s)}}^{\alpha_{n}}=\tilde{K}_{n}^{\alpha_{n}} .
\end{aligned}
$$

This completes the proof of inequality (1). This inequality gives that the operator $\sigma_{*}^{\alpha}$ is also of weak type $\left(L^{1}, L^{1}\right)$ since 


$$
\lambda\left(\sigma_{*}^{\alpha} f>2 C_{K} \delta\right) \leq \lambda\left(\tilde{\sigma}_{*}^{\alpha}|f|>2 C_{K} \delta\right) \leq C_{K} \frac{\||f|\|_{1}}{\delta}=C_{K} \frac{\|f\|_{1}}{\delta} .
$$

This completes the proof of Lemma 6.

Proof of Theorem 1. Let $P \in \mathbf{P}$ be a polynomial where $P(x)=\sum_{i=0}^{2^{k}-1} c_{i} \omega_{i}$. Then for all natural number $n \geq 2^{k}, n \in \mathbb{N}_{\alpha, K}$ we have that $S_{n} P \equiv P$. Consequently, the statement $\sigma_{n}^{\alpha_{n}} P \longrightarrow P$ holds everywhere (of course not only for restricted $n \in$ $\left.\mathbb{N}_{\alpha, K}\right)$. Now, let $\epsilon, \delta>0, f \in L^{1}$. Let $P \in \mathbf{P}$ be a polynomial such that $\|f-P\|_{1}<\delta$. Then

$$
\begin{aligned}
& \lambda\left(\overline{\lim }_{n \in \mathbb{N}_{\alpha, K}}\left|\sigma_{n}^{\alpha_{n}} f-f\right|>\epsilon\right)
\end{aligned}
$$

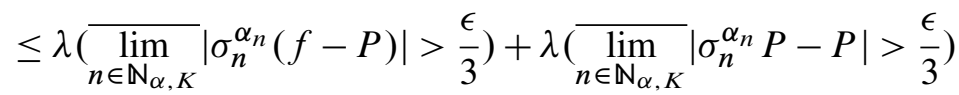

$$
\begin{aligned}
& +\lambda\left(\overline{\lim }_{n \in \mathbb{N}_{\alpha, K}}|P-f|>\frac{\epsilon}{3}\right) \\
& \leq \lambda\left(\sup _{n \in \mathbb{N}_{\alpha, K}}\left|\sigma_{n}^{\alpha_{n}}(f-P)\right|>\frac{\epsilon}{3}\right)+0+\frac{3}{\epsilon}\|P-f\|_{1} \leq C_{K}\|P-f\|_{1} \frac{3}{\epsilon} \leq \frac{C_{K}}{\epsilon} \delta
\end{aligned}
$$

because $\sigma_{*}^{\alpha}$ is of weak type $\left(L^{1}, L^{1}\right)$ (with any fixed $K>0$ ). This holds for all $\delta>0$. That is, for an arbitrary $\epsilon>0$ we have

$$
\lambda\left(\overline{\lim }_{n \in \mathbb{N}_{\alpha, K}}\left|\sigma_{n}^{\alpha_{n}} f-f\right|>\epsilon\right)=0
$$

and consequently we also have

$$
\lambda\left(\varlimsup_{n \in \mathbb{N}_{\alpha, K}}\left|\sigma_{n}^{\alpha_{n}} f-f\right|>0\right)=0 .
$$

This finally gives

$$
\begin{aligned}
& \varlimsup_{n \in \mathbb{N}_{\alpha, K}}\left|\sigma_{n}^{\alpha_{n}} f-f\right|=0 \text { a.e, } \\
& \sigma_{n}^{\alpha_{n}} f \longrightarrow f \text { a.e. } \quad\left(n \in \mathbb{N}_{\alpha, K}\right) .
\end{aligned}
$$

This completes the proof of Theorem 1 .

Proof of Theorem 2. Inequality (1), Lemma 5 and Lemma 6 by the interpolation theorem of Marcinkiewicz [9] give that the operator $\sigma_{*}^{\alpha}$ is of type $\left(L^{p}, L^{p}\right)$ for all $1<p \leq \infty$. In the sequel we prove that operator $\tilde{\sigma}_{*}^{\alpha} f=\sup _{n \in \mathbb{N}_{\alpha, K}}\left|f * \tilde{K}_{n}^{\alpha}\right|$ is of type $(H, L)$.

Let $a$ be an atom $\left(a \neq 1\right.$ can be supposed ), supp $a \subset I_{k}(x),\|a\|_{\infty} \leq 2^{k}$ for some $k \in N$ and $x \in I$. Then, $n<2^{k}, n \in \mathbb{N}_{\alpha, K}$ implies $a * \tilde{K}_{n}^{\alpha}=0$ because $\tilde{K}_{n}^{\alpha}$ is $\mathcal{A}_{k}$ measurable for $n<2^{k}$ and $\int_{I_{k}(x)} a(t) d \lambda(t)=0$. That is,

$$
\tilde{\sigma}_{*}^{\alpha} a=\sup _{\mathbb{N}_{\alpha, K} \ni n \geq 2^{k}}\left|\tilde{\sigma}_{n}^{\alpha_{n}} f\right| \text {. }
$$


By the help Lemma 4 we have

$$
\begin{aligned}
& \int_{I \backslash I_{k}(x)} \tilde{\sigma}_{*}^{\alpha} a d \lambda=\int_{I \backslash I_{k}(x)} \sup _{\mathbb{N}_{\alpha, K} \ni n \geq 2^{k}}\left|\int_{I_{k}(x)} a(y) \tilde{K}_{n}^{\alpha_{n}}(z+y) d \lambda(y)\right| d \lambda(z) \\
& \leq C_{K} \int_{I_{k}(x)}|a(y)| d \lambda(y) \\
& \leq C_{K}\|a\|_{1} \\
& \leq C_{K} .
\end{aligned}
$$

Since the operator $\tilde{\sigma}_{*}^{\alpha}$ is of type $\left(L^{2}, L^{2}\right)$ (i.e $\left\|\tilde{\sigma}_{*}^{\alpha} f\right\|_{2} \leq C_{K}\|f\|_{2}$ for all $\left.f \in L^{2}(I)\right)$, then we have

$$
\begin{aligned}
& \left\|\tilde{\sigma}_{*}^{\alpha} a\right\|_{1}=\int_{I \backslash I_{k}(x)} \tilde{\sigma}_{*}^{\alpha} a+\int_{I_{k}(x)} \tilde{\sigma}_{*}^{\alpha} a \\
& \leq C_{K}+\left|I_{k}(x)\right|^{\frac{1}{2}}\left\|\tilde{\sigma}_{*}^{\alpha} a\right\|_{2} \\
& \leq C_{K}+C_{K} 2^{\frac{-k}{2}}\|a\|_{2} \\
& \leq C_{K}+C_{K} 2^{\frac{-k}{2}} 2^{\frac{k}{2}} \\
& \leq C_{K} .
\end{aligned}
$$

That is $\left\|\tilde{\sigma}_{*}^{\alpha} a\right\|_{1} \leq C_{K}$ and consequently the $\sigma$-sublinearity of $\tilde{\sigma}_{*}^{\alpha}$ gives

$$
\begin{aligned}
& \left\|\tilde{\sigma}_{*}^{\alpha} f\right\|_{1} \leq \sum_{i=0}^{\infty}\left|\lambda_{i}\right|\left\|\tilde{\sigma}_{*}^{\alpha} a_{i}\right\|_{1} \\
& \leq C_{K} \sum_{i=0}^{\infty}\left|\lambda_{i}\right| \\
& \leq C_{K}\|f\|_{H}
\end{aligned}
$$

for all $\sum_{i=0}^{\infty} \lambda_{i} a_{i} \in H$. That is, the operator $\tilde{\sigma}_{*}^{\alpha}$ is of type $(H, L)$. This by inequality (1) and then by $\left\|\sigma_{*}^{\alpha} f\right\|_{1} \leq\left\|\tilde{\sigma}_{*}^{\alpha}|f|\right\|_{1} \leq C_{K}\||f|\|_{H}$ completes the proof of Theorem 2.

\section{REFERENCES}

[1] T. Akhobadze, "On the convergence of generalized Cesáro means of trigonometric Fourier series. I.” Acta Math. Hung., vol. 115, no. 1-2, pp. 59-78, 2007, doi: https://doi.org/10.1007/s10474007-5214-7.

[2] T. Akhobadze, "On the convergence of generalized Cesáro means of trigonometric Fourier series." Bulletin of TICMI, vol. 18, no. 1, pp. 75-84, 2014.

[3] N. Fine, "Cesàro summability of Walsh-Fourier series," Proc. Nat. Acad. Sci. USA, vol. 41, pp. 588-591, 1955, doi: https://doi.org/10.1073/pnas.41.8.588.

[4] S. Fridli, "On the rate of convergence of cesàro means of walsh-fourier series," J. of Approx. Theory, vol. 76, no. 1, pp. 31-53, 1994, doi: https://doi.org/10.1006/jath.1994.1003. 
[5] N. Fujii, "A maximal inequality for $h^{1}$ functions on the generalized walsh-paley group," Proc. Amer. Math. Soc., vol. 77, pp. 111-116, 1979, doi: https://doi.org/10.1090/S0002-9939-19790539641-9.

[6] G. Gát, "On (c,1) summability for vilenkin-like systems," Stud. Math., vol. 144, no. 2, pp. 101120, 2001, doi: 10.4064/sm144-2-1.

[7] U. Goginava, "Approximation properties of (c, $\alpha)$ means of double walsh-fourier series," Analysis in Theory and Applications, vol. 20, no. 1, pp. 77-98, 2004, doi: https://doi.org/10.1007/BF02835261.

[8] E. Hewitt and K. Ross, Abstract Harmonic Analysis I. Heidelberg: Springer-Verlag, 1963.

[9] F. Schipp, W. Wade, P. Simon, and J. Pál, Walsh series,"An Introduction to dyadic harmonic analysis”. Bristol and New York: Adam Hilger, 1990.

[10] M. Taibleson, Fourier Analysis on Local Fields. Princeton, N.J.: Princeton Univ. Press., 1975.

[11] F. Weisz, “(C, $\alpha)$ summability of Walsh-Fourier series," Analysis Mathematica, vol. 27, pp. 141$155,2001$.

[12] A. Zygmund, Trigonometric Series. Cambridge: University Press, 1959.

Authors' addresses

Anas Ahmad Abu Joudeh

Institute of Mathematics, University of Debrecen, H-4010 Debrecen, Pf. 12, Hungary

E-mail address: anas.abujoudeh@mailbox.unideb.edu.hu, mr_anas_judeh@yahoo.com

\section{György Gát}

Institute of Mathematics, University of Debrecen, H-4010 Debrecen, Pf. 12, Hungary

E-mail address: gat.gyorgyescience.unideb.hu 